\title{
On Wilkinson's Method of treating the Nine-Points Circle, with Generalizations.
}

\author{
By R. F. Muirhead, M.A., D.Sc.
}

(Read 12th December 1909. Received 25th April 1910).

Ever since I first became acquainted with Wilkinson's method of establishing the existence of the nine-points circle of a triangle (see Mackay's " Euclid," Appendix to Bk. IV., Prop. 2, the lettering of which I have followed in the first three sections of this paper), its simple and fundamental character has pleased me. I propose to point out first that this method yields probably the most elementary proof of the concurrence of the perpendiculars from the vertices, and then, after restating the investigation of the nine-points circle, to sketch some generalizations.

$\$ 1$.

The method depends on the following propositions, which are either contained in Euclid's first book or are easy and wellknown deductions from it. I shall use the symbol $\equiv$ to denote "is equal and parallel to, and has the same sense as."

Prop. (i) If $A B \equiv C D$ then $A C \equiv B D$.

" (ii) If $A B \| C D$ and $A C \| B D$ then $A B \equiv C D$ and $A C \equiv B D$.

"(iii) If $A B \equiv C D$ then $A D$ and $B C$ bisect one another.

"(iv) If $A D$ and $C D$ bisect one another, then $A B \equiv C D$ and $\mathrm{AC} \equiv \mathrm{BD}$.

" (v) If $\mathrm{D}$ is the mid point of $\mathrm{AB}$ and $\mathrm{E}$ that of $\mathrm{AC}$, then $\mathrm{BC} \equiv 2 \mathrm{DE}$.

"(vi) If $\mathrm{D}$ is the mid point of $\mathrm{AB}$ and $\mathrm{DE} \| \mathrm{BC}, \mathrm{E}$ being in $A C$, then $\mathrm{E}$ is the mid point of $\mathrm{AC}$.

, (vii) If $A B C D$ is a rectangle, $A C$ and $B D$ are equal and bisect one another.

"(viii) If $A C$ and $B D$ are equal and bisect one another, $A B C D$ is a rectangle. 
Let $\mathrm{ABC}$ be a triangle (Fig. 1). Let the perpendiculars $\mathrm{BY}, \mathrm{C}$. let fall from $B$ and $C$ on $A C$ and $A B$ intersect in $O$.

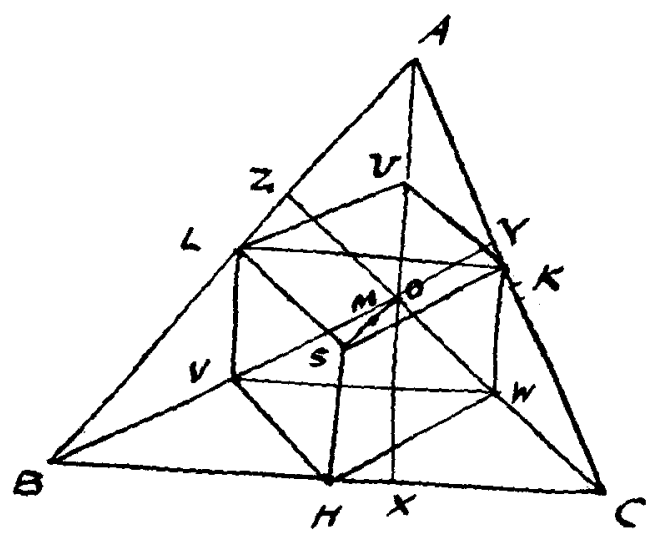

Fig. 1.

Let $\mathrm{AO}$ meet $\mathrm{BC}$ in $\mathrm{X}$ : we have to prove that $\mathrm{AX}$ is perpendicular to $\mathrm{BC}$.

Let $B, K, L$, be the mid points of $B C, C A, A B$ respectively.

$$
\text { "U, } \mathrm{V}, \mathrm{W}, \quad, \quad, \quad \mathrm{OA}, \mathrm{OB}, \mathrm{OC} \text { " }
$$

By (v) $U V \equiv \frac{1}{2} A B \equiv K H$.

$$
\mathrm{VH} \equiv \frac{1}{2} \mathrm{OC} \equiv \mathrm{UK} \text {. }
$$

Hence UVHK is a parallelogram whose sides are parallel to $\mathrm{AB}$ and $\mathrm{CO}$, which are at right angles. Hence UVHK is a rectangle.

Hence by (vii) $\mathrm{HU}$ and $\mathrm{KV}$ are equal, and bisect one another.

Similarly WULH is a rectangle, and $\mathrm{HU}$ and $L W$ are equal, and bisect one another.

Hence $\mathrm{KV}$ and $\mathrm{LW}$ are equal, and bisect one another.

Hence $K L V W$ is a rectangle by (viii).

Hence $\mathrm{BC}$ and $\mathrm{AX}$, which by ( $\nabla$ ) are parallel to its sides, are mutually perpendicular.

Thus the three perpendiculars from $A, B, C$ on the opposite sides are concurrent. Q. E. D. 
Cor. 1. By (vii) $\mathrm{HU}, \mathrm{KV}, \mathrm{LW}$ are all equal and bisect one another in the same point $\mathrm{M}$, which is therefore the centre of a circle passing through UVWHKL.

$\mathrm{By}$ (vii) this circle also passes through $\mathrm{XYZ}$, since $\mathrm{BXU}$ form two sides of a rectangle whose mid point is $M$.

Cor. 2. By (v) OVHW, OWKU, OULV are parallelograms, and if we complete the parallelogram LVHS, then

$$
\mathrm{SH} \equiv \mathrm{LV} \equiv \mathrm{UO} \equiv \mathrm{KW} \text { by (ii). }
$$

Hence by (iii) $\mathbf{M}$ is the mid point of So.

By (i) $\mathrm{SK} \equiv \mathrm{HW} \equiv \mathrm{VO} \equiv \mathrm{LU}$ $\mathrm{SL} \equiv \mathrm{KU} \equiv \mathrm{WO} \equiv \mathrm{HV}$.

Hence $\quad \mathrm{S}$ is the circumcentre. Also $\quad \mathrm{AO} \equiv 2 \mathrm{UO} \equiv \mathrm{SH}$, etc.

Cor. 3. The triangles $\mathrm{UVW}$ and $\mathrm{ABC}$ are homothetic, $\mathrm{O}$ being the homothetic centre, and $M$ and $S$ corresponding points, the ratio of linear dimensions being $1: 2$.

Hence OX produced meets the circumcircle of $A B C$ at a distance from $\mathrm{O}=2 \mathrm{OX}$.

Cor. 4. If $\mathrm{AH}$ meets $\mathrm{SO}$ in $\mathrm{G}$, since $\mathrm{AO}=2 \mathrm{SH}, \mathrm{OG}=2 \mathrm{GS}$. Similarly BK and CL cut OS in the same point $G$, which is therefore the point of concurrence of the medians.

$\$ 2$.

The generalization from the nine-points circle to the ninepoints conic worked out by $\mathrm{Dr}$ P. Pinkerton in his paper in Vol. XXIV. can be established with great ease by Wilkinson's method.

Let $S$ be any point in the plane of $A B C$, and $H, K, L$, as before, the mid points of $\mathrm{BC}, \mathrm{CA}, \mathrm{AB}$.

Let $\mathrm{CZ} \|$ to $\mathrm{SL}$ and $\mathrm{BY} \|$ to $\mathrm{SK}$ meet $\mathrm{AB}$ in $\mathrm{Z}, \mathrm{CA}$ in $\mathrm{Y}$, and each other in $O$.

Let $\mathrm{AO}$ meet $\mathrm{BC}$ in $\mathrm{X}$.

As before, let $U, V, W$ be the mid points of $O A, O B, O C$.

Obviously OVHW, OWKU, OULV are parallelograms, and $\mathrm{SK} \|$ to $\mathrm{OB} \|$ to $\mathrm{UL}\}$. SLUK is a parallelogram.

$\Rightarrow \quad \mathrm{SL} \|$ to $\mathrm{OC} \|$ to $\mathrm{UK}$
$\therefore \quad \mathrm{SK} \equiv \mathrm{LU} \equiv \mathrm{VO} \equiv \mathrm{HW}$

$\therefore$ SHWK and similarly SHVL are parallelograms.

Thus $\mathrm{SH} \equiv \mathrm{KW} \equiv \mathrm{UO}$, i.e. the lines through $\mathrm{A}, \mathrm{B}, \mathrm{C}$ parallel to SH, SK, SL are concurrent. 
We have also SO, UH, VK, LW bisecting one another in the same point $M$.

Now UVWMO and ABCSO are homothetic, the ratio of their linear dimensions being $1: 2$.

Hence if any figure be described about $\mathrm{ABC}$, a homothetic figure of half of its linear dimensions can be described about UVW.

If the circumscribing figure be a central curve of any sort, with $\mathrm{S}$ as centre, then the homothetic curve round UVW will have $\mathrm{M}$ as centre, and will therefore pass through $\mathrm{HKL}$.

If the central curve be a conic, so that supplementary chords are parallel to conjugate diameters and conversely, then $\mathrm{XYZ}$ will also lie on the conic UVWHKL. For $\mathrm{SH}$ and $\mathrm{BC}$ being conjugate directions as to the conic through $\mathrm{ABC}$, they are so as to the conic UVWHKL. Thus HX, UX, drawn through the extremities of a diameter $\mathrm{HU}$, are parallel to a pair of conjugate diameters, and therefore $\mathrm{X}$ lies on the conic. Similarly $\mathrm{Y}$ and $\mathrm{Z}$.

Every line from $O$ to a point of the smaller conic meets the larger at double the distance. We note the special cases of the points $\mathrm{X}, \mathrm{Y}, \mathrm{Z}, \mathrm{H}, \mathrm{K}, \mathrm{L}$.

Thus the properties of the nine-points conic investigated by Dr Pinkerton in his paper in Vol. XXIV. have been arrived at by Wilkinson's method, with the additional aid of the elements of the theory of homothesis, and the proposition about supplementary chords and conjugate diameters of a conic.

$$
\$ 3 .
$$

The whole of the preceding theory can be generalized further by first generalizing the fundamental propositions (i)...(viii) on which it is based.

We define certain phrases.

Given a fixed line $\alpha$, if $\mathrm{H}$ is the harmonic conjugate as to $\mathrm{BC}$ of its intersection with $a$, we shall say that $\mathrm{H}$ "bisects $\mathrm{BC}$ as to $a . "$

If $\mathrm{AB}$ and $\mathrm{CD}$ are concurrent in $\alpha$ and also $\mathrm{AC}$ and $\mathrm{BD}$, we shall say $\mathrm{AB} \equiv \mathrm{CD}$ and $\mathrm{AC} \equiv \mathrm{BD}$.

Hence the symbol $\equiv$ refers only to segments of lines which are concurrent in $a$.

When $a$ is the line at infinity, this meaning reduces to that previously assigned to the symbol $\equiv$, and the fundamental 
property. "If $\mathrm{AB} \equiv \mathrm{CD}$ and $\mathrm{CD} \equiv \mathrm{EF}$, then $\mathrm{AB} \equiv \mathrm{EF}$," required to make the preceding proofs apply, is obviously a special case of Desargues' Theorem applied to the perspective triangles ACE, BDF.

In place of the word parallel we shall in the generalized theory substitute concurrent in $\alpha$.

For our fundamental propositions (i)-(viii) in the generalized theory we have :

(i) and (ii) are immediate results of the extended definitions.

(iii) If $\mathrm{AB} \equiv \mathrm{CD}$, then $\mathrm{AD}$ and $\mathrm{BC}$ " bisect one another as to a." This is the harmonic property of the complete quadrilateral.

(iv) If $\mathrm{AD}$ and $\mathrm{BC}$ "bisect one another as to $a$," then $\mathrm{AB} \equiv \mathrm{CD}$ and $\mathrm{AC} \equiv \mathrm{BD}$.

(v) If $\mathrm{D}$ "bisects $\mathrm{AB}$ as to $\alpha$ " and $\mathrm{E}$ "bisects $\mathrm{AC}$ as to $\alpha$," then $\mathrm{BC} \equiv 2 \mathrm{DE}$

(iv) and (v) are cases of the proposition that if two harmonic ranges have one pair of corresponding points coincident, the joins of the other corresponding pairs are concurrent.

(vi) If $\mathrm{D}$ "bisects $\mathrm{AB}$ as to $\alpha$ " and $\mathrm{DE}$ and $\mathrm{BC}$ are concurrent in a, $\mathrm{E}$ being in $\mathrm{AC}$, then $\mathrm{E}$ " bisects $\mathrm{AC}$ as to $a . "$ This is an application of the theorem that if one transversal of a pencil gives a harmonic range, so does any other.

(vii) and (viii) do not occur in the generalized theory.

The generalised theory begins with $\mathrm{H}, \mathrm{K}, \mathrm{L}$ " bisecting as to $a$ " the sides $\mathrm{BC}, \mathrm{CA}, \mathrm{AB}$, and $\mathrm{BY}, \mathrm{CZ}$ concurrent in a with $\mathrm{SK}, \mathrm{SL}$ respectively, $\mathrm{S}$ being any point. $\mathrm{O}$ is the intersection of $\mathrm{BY}, \mathrm{CZ}$. U, V, W "bisect" OA, OB, OC "as to a." (See Fig. 2.)

$\mathrm{LU}$ and $\mathrm{BO}$ are concurrent in $\alpha$ by (v).

$\therefore \mathrm{LU}$ and SK are concurrent in $a$.

Similarly SL and UK , , , ",

$\therefore \mathrm{SK} \equiv \mathrm{LU} \equiv \mathrm{VO} \equiv \mathrm{HW}$.

$\therefore \mathrm{SH} \equiv \mathrm{KW} \equiv \mathrm{OU}$.

$\therefore \mathrm{AX}$ and SH are concurrent in a.

It follows that:

If lines be drawn through $\mathrm{A}, \mathrm{B}, \mathrm{C}$ respectively, concurrent in $a$ with $\mathrm{SH}, \mathrm{SK}, \mathrm{SL}$ respectively, where $H, \mathrm{~K}, \mathrm{~L}$ " bisect " $\mathrm{BC}, \mathrm{CA}, \mathrm{AB}$ "as to $a$," these lines will be concurrent in a point $O$. 


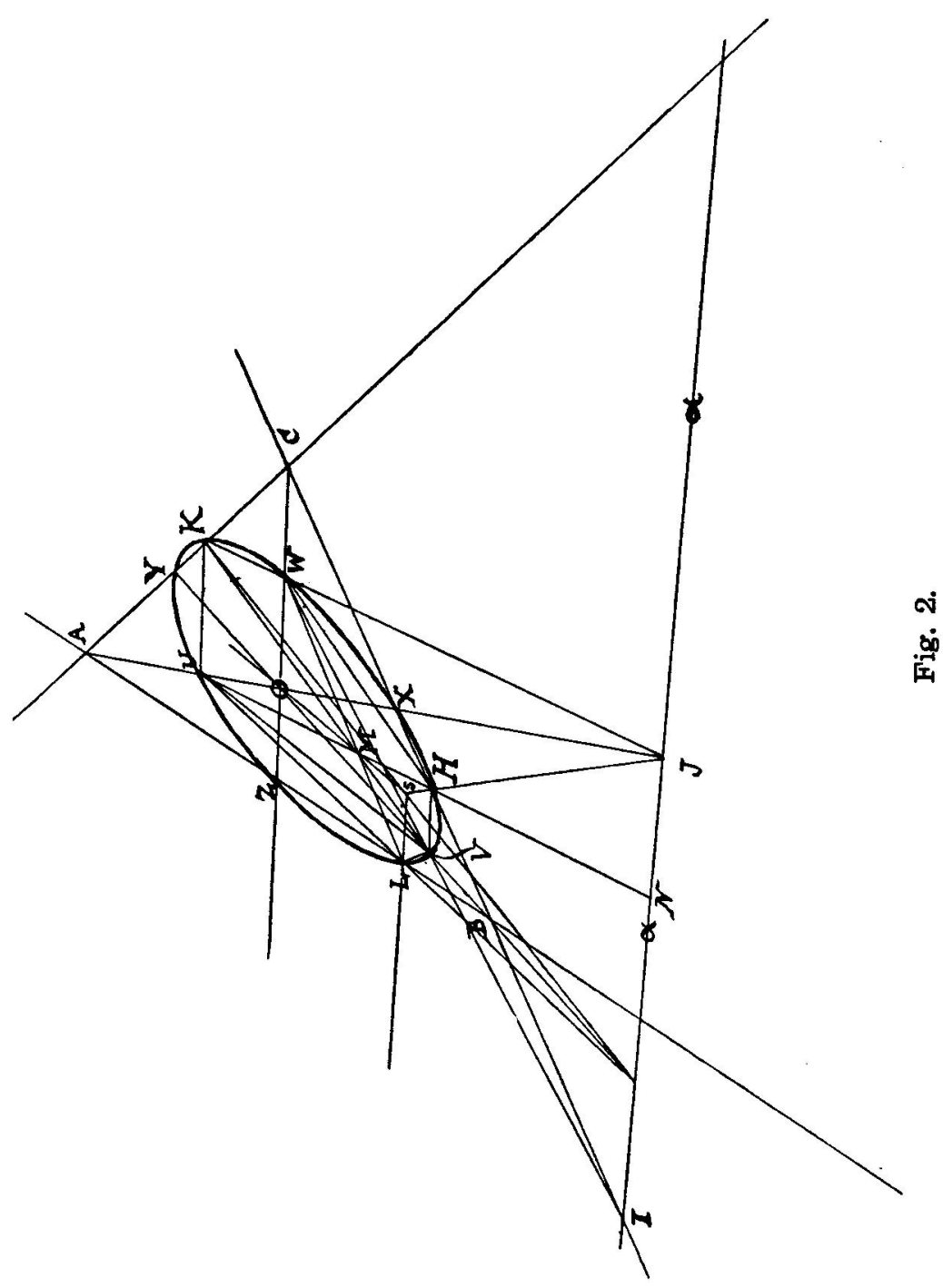


The eight points $\mathrm{O}, \mathrm{S}, \mathrm{U}, \mathrm{V}, \mathrm{W}, \mathrm{H}, \mathrm{K}, \mathrm{L}$, are such that $\mathrm{OS}, \mathrm{HU}, \mathrm{KV}, \mathrm{LW}$ all "bisect one another as to $a$ " in the same point $M$.

To get the nine-points conic in its generalised form, we must give the appropriate generalization of the theory of homothesis, and of the property that supplementary chords have conjugate directions.

If every point $P$ of a figure $\phi$ be joined to a fixed point $M$, and $P M$ be divided by $P^{\prime}$ and the fixed line $\alpha$, in a given anharmonic ratio $\lambda$, then $\phi^{\prime}$, the assemblage of points $P^{\prime}$ may be said to be "homothetic as to $a$ " with $\phi$, having $\mathbf{M}$ as homothetic centre, and $\lambda$ as homothetic ratio, for lines joining corresponding points of $\phi$ and $\phi^{\prime}$ will obviously be concurrent in $\alpha$.

(Here $\phi$ and ' $\phi^{\prime}$ are in perspective, the perspective centre being $M$ and the perspective axis $\alpha$ ).

It follows that if $P Q R$ in $\phi$ be collinear, then $P^{\prime} Q^{\prime} R^{\prime}$ in $\phi^{\prime}$ will also be collinear. Hence by Pascal's Theorem, if six points in $\phi$ are on a conic, the corresponding six points in $\phi^{\prime}$ are also on a conic, so that every figure "homothetic as to $a$ " with a conic is itself a conic.

If then $\Gamma$ be a conic round $\mathrm{ABC}$, and $\mathrm{S}$ the pole of $a$ as to $\Gamma$, and if $\gamma$ be a conic homothetic with $\Gamma$ as to $\alpha$, with $O$ as homothetic centre and homothetic ratio $=-1$, then $\gamma$ will pass through UVW, and M will be the pole of $a$ as to $\gamma$.

Hence if $\mathrm{HU}$ meets $\alpha$ in $\mathrm{N}, \mathrm{HU}$ is divided harmonically by $M$ and $N$. Hence, $U$ being on $\gamma$, so is $H$. Similarly $K$ and $L$ are on $\gamma$.

The generalised form of the proposition that supplementary chords of a conic are in conjugate directions is this :

Two points on $\alpha$ are said to be conjugate as to the conic $\gamma$ if each lies on the polar of the other.

If $P Q$ is a chord passing through $M$ the pole of a (see Fig. 3), and $R$ is any other point on $\alpha$, then $P R$ and $Q R$ may be said to be chords of $\gamma$ which are "supplementary as to $\alpha . "$

Such chords intersect $\alpha$ in points $q$ and $p$, which are conjugate.

For if $a$ and $\mathrm{PQ}$ intersect in $r$ and $q \mathrm{M}$ and $\mathrm{QR}$ meet in $\mathrm{T}$, then $\{\mathrm{QRT} p\}=\{\mathrm{QPM} r\}=-1$.

$\therefore \mathrm{T}$ is on the polar of $p$. So is $\mathrm{M}, \therefore q \mathrm{M}$ is the polar of $p$.

Thus if PR and QR are "supplementary" chords of $\gamma$ "as to $a$," then $P R$ and $Q R$ meet $a$ in conjugate points. 


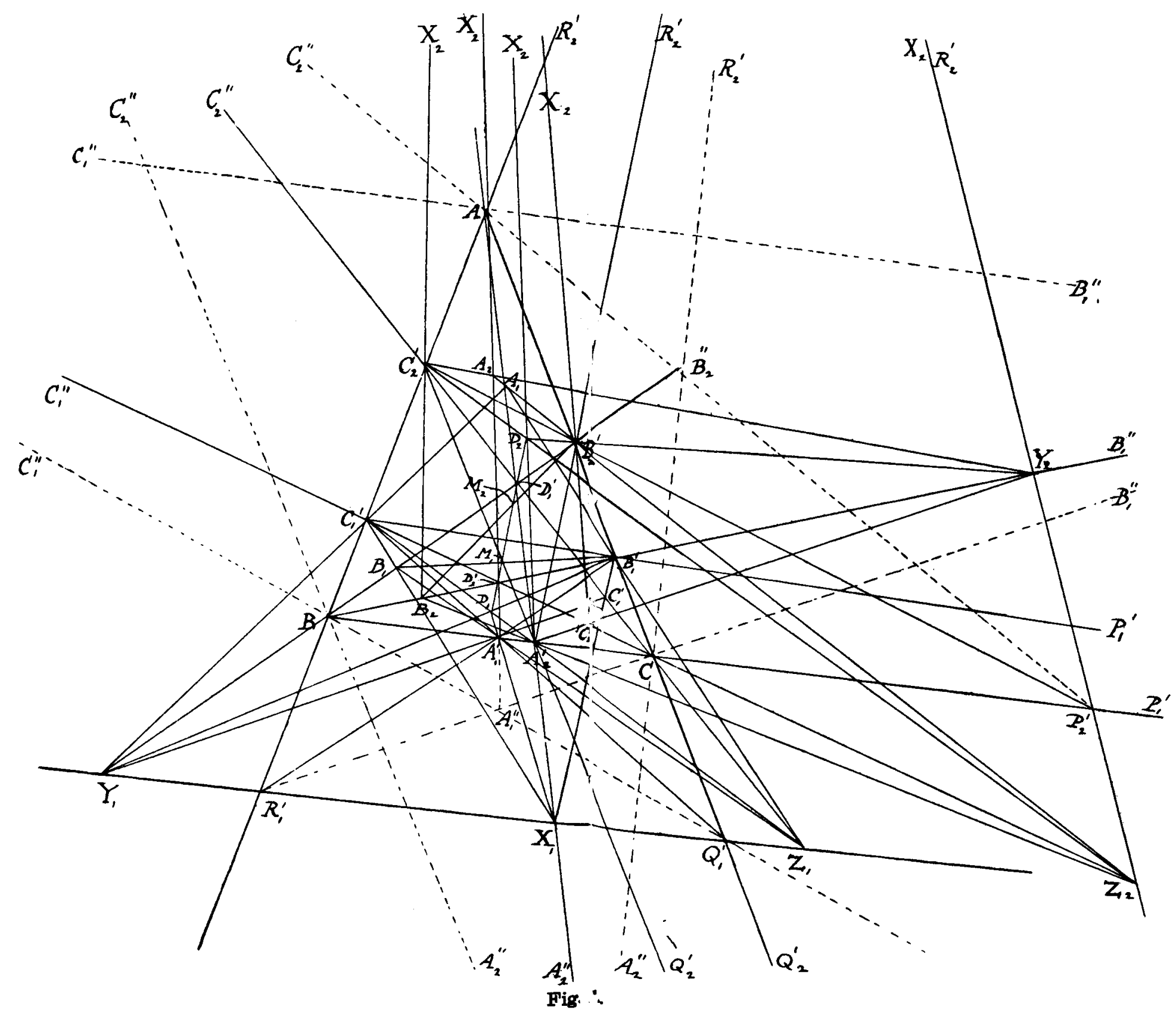


Conversely, if through the extremities of a chord of $\gamma$ through $M$ lines be drawn to conjugate points on a, these will meet in a point $R$ on the conic.

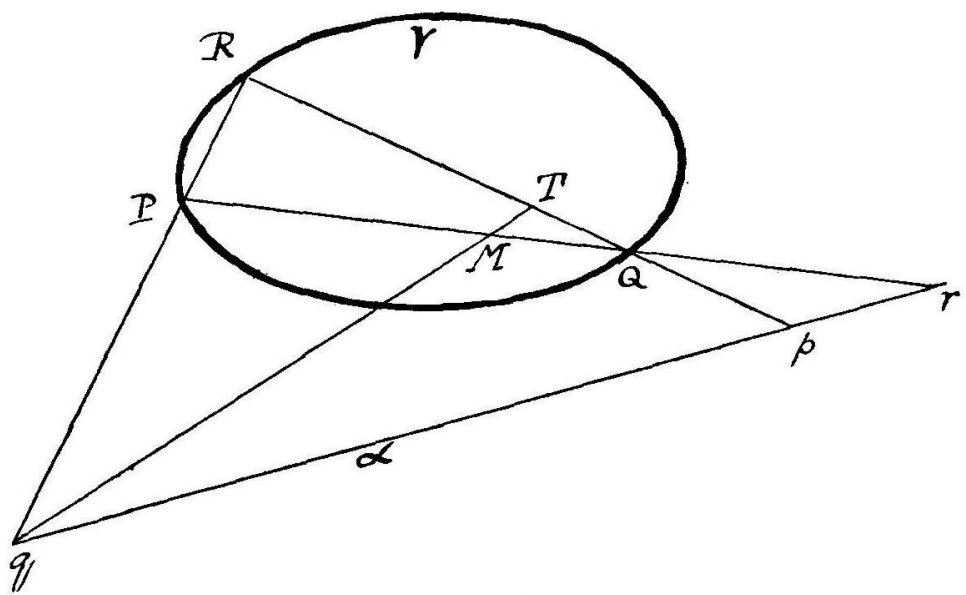

Fig. 3.

Returning to Fig. $2, \mathrm{HX}$ and $\mathrm{VW}$ are concurrent in a point $\mathrm{I}$ of $\alpha$, and $U X$ and $K W$ in a point $J$ of $a$.

But $\mathrm{KW}$ and $\mathrm{VW}$ are "supplementary" chords of $\gamma$ "as to a." Hence I and J are "conjugate points as to $\gamma . "$

Hence HX and UX are "supplementary" chords of $\gamma$ as to a, i.e. $\mathrm{X}$ lies on the conic $\gamma$. So do $\mathrm{Y}$ and $\mathrm{Z}$.

Thus for every point $S$ and line $a$ associated with the triangle ABC there exists a generalized nine-points conic $\gamma$ which passes through U, V, W, H, K, L, X, Y, Z, when H, K, L "bisect" BC, CA, $\mathrm{AB}$, "as to $\alpha$," and $\mathrm{AX}, \mathrm{BY}, \mathrm{CZ}$ are concurrent in $\alpha$ with $\mathrm{SH}, \mathrm{SK}$, SL respectively, and $U, V, W$ " bisect as to $a$ " the segments $A O$, $\mathrm{BO}, \mathrm{CO}, \mathrm{O}$ being the point of concurrence of $\mathrm{AX}, \mathrm{BY}, \mathrm{CZ}$.

And the pole $M$ of $\alpha$ as to this conic "bisects" SO "as to $a$," and $\mathrm{S}$ is the pole of $a$ of a conic round $\mathrm{ABC}$ which is "homothetic as to $a$ " with $\gamma, \mathrm{O}$ being the homothetic centre.

By sending $\alpha$ to infinity this reduces to the ordinary theory of the nine-points conic. 
$\$ 4$.

We may pursue this line of investigation a little further, as follows. For the sake of symmetry we will change the lettering to some extent, as may be seen by comparing Fig. 4 with Fig. 2. $\mathrm{P}_{1}{ }^{\prime} \mathrm{Q}_{1} \mathrm{R}_{1}{ }^{\prime}$ is a transversal of the triangle $\mathrm{ABC}$, forming with it a complete quadrilateral. (See Fig. 4).

$A_{1}^{\prime \prime} B_{1}^{\prime \prime} C_{1}$ " is its diagonal triangle.

$\mathrm{AA}_{1}{ }^{\prime \prime}, \mathrm{BB}_{1}{ }^{\prime \prime}, \mathrm{CC}_{1}^{\prime \prime}$, concur in $\mathrm{D}_{2}^{\prime}$, and cut $\mathrm{BC}, \mathrm{CA}, \mathrm{AB}$ in $A_{1}^{\prime}, B_{1}^{\prime}, C_{1}^{\prime}$.

Again $P_{2}^{\prime} Q_{2}^{\prime} R_{2}^{\prime}$ is another transversal of $A B C$, giving another complete quadrilateral, which, treated like the preceding one, gives rise to points $\mathrm{A}_{2}{ }^{\prime \prime} \mathrm{B}_{2}{ }^{\prime \prime} \mathrm{C}_{2}{ }^{\prime \prime} \mathrm{A}_{2}{ }^{\prime} \mathrm{B}_{2}{ }^{\prime} \mathrm{C}_{2}{ }^{\prime} \mathrm{D}_{1}$.

We then combine the quadrilaterals.

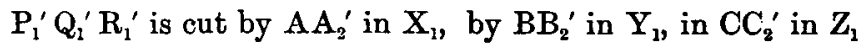
$\mathrm{Y}_{1} \mathrm{C}_{1}^{\prime}, \mathrm{Z}_{1} \mathrm{~B}_{1}{ }^{\prime}$ and $A \mathrm{D}_{1}{ }^{\prime}$ concur in $\mathrm{A}_{1}$

$\mathrm{Z}_{1} \mathrm{~A}_{1}^{\prime}, \mathrm{X}_{1} \mathrm{C}_{1}^{\prime}$ and $\mathrm{BJ}_{1}^{\prime} \quad " \quad, \mathrm{~B}_{1}$

$\mathrm{X}_{1} \mathrm{~B}_{1}^{\prime}, \mathrm{Y}_{1} \mathrm{~A}_{1}^{\prime}$ and $\mathrm{CD}_{1}^{\prime} \quad, \quad " \mathrm{C}_{1}$.

Similarly $\mathrm{Y}_{2} \mathrm{C}_{2}^{\prime}, Z_{2} \mathrm{~B}_{2}^{\prime}$, and $\mathrm{AD}_{2}^{\prime}$ concur in $\mathrm{A}_{2}$, etc.

$\mathrm{X}_{1} \mathrm{~A}_{1}^{\prime}, \mathrm{Y}_{1} \mathrm{~B}_{1}^{\prime}, \mathrm{Z}_{1} \mathrm{C}_{1}{ }^{\prime}$ concur in $\mathrm{D}_{1}$

$\mathrm{X}_{2} \mathrm{~A}_{2}^{\prime}, \mathrm{Y}_{2} \mathrm{~B}_{2}^{\prime}, \mathrm{Z}_{2} \mathrm{C}_{2}^{\prime} \quad, \quad, \mathrm{D}_{2}$

$\mathrm{D}_{1} \mathrm{D}_{2}^{\prime} \mathrm{D}_{1}^{\prime} \mathrm{D}_{2}$ are collinear.

$A_{1} A_{1}^{\prime}, B_{1} B_{1}^{\prime}, C_{1} C_{1}^{\prime}$ concur in $M_{1}$

$\mathrm{A}_{2} \mathrm{~A}_{2}^{\prime}, \mathrm{B}_{2} \mathrm{~B}_{2}^{\prime}, \mathrm{C}_{2} \mathrm{C}_{2}^{\prime} \quad, \quad, \mathrm{M}_{2}$.

A twelve-points conic passes through

$$
A_{1} B_{1} C_{1} A_{1}^{\prime} B_{1}^{\prime} C_{1}^{\prime} A_{2} B_{2} C_{2} A_{2}^{\prime} B_{2}^{\prime} C_{2}^{\prime} \text {. }
$$

With respect to this conic, $M_{1}$ is the pole of $P_{1}^{\prime} Q_{1}^{\prime} R_{1}^{\prime}$, and $M_{2}$ that of $\mathrm{P}_{2}^{\prime} \mathrm{Q}_{2}^{\prime} \mathrm{R}_{2}^{\prime}$.

$D_{1}$ is pole of $P_{1}^{\prime} Q_{1}^{\prime} R_{1}^{\prime}$ as to the circumconic, with $D_{1}^{\prime}$ as centre, and $P_{1}^{\prime} Q_{1}^{\prime} R_{1}^{\prime}$ as axis of perspective.

$\mathrm{D}_{2}$ is pole of $\mathrm{P}_{2}^{\prime} \mathrm{Q}_{2}^{\prime} R_{2}^{\prime}$ as to a circumconic of $\mathrm{ABC}$ in perspective with the twelve-points conic, and having $\mathrm{D}_{2}^{\prime}$ as centre of perspective and $P_{2}^{\prime} Q_{2}^{\prime} R_{2}^{\prime}$ as axis.

If we send $P_{1}^{\prime} Q_{1}^{\prime} R_{1}^{\prime}$ off to infinity, $A_{1}{ }^{\prime} B_{1}^{\prime} C_{1}^{\prime}$ become the mid points of the sides of the triangle $\mathrm{ABC}$, and we get the special twelve-points conic, which again becomes a circle, when for the transversal $P_{2}^{\prime} Q_{2}^{\prime} R_{2}^{\prime}$ we take the "orthic axis" of the triangle $\mathrm{ABC}$. 
Similarly, if $P_{2}^{\prime} Q_{2}^{\prime} R_{2}^{\prime}$ be sent to infinity, and the "orthic axis" of $\mathrm{ABC}$ is taken for $\mathrm{P}_{1}^{\prime} \mathrm{Q}_{1}^{\prime} \mathrm{R}_{1}^{\prime}$, then $\mathrm{AA}_{2}^{\prime}, \mathrm{BB}_{2}^{\prime}, \mathrm{CC}_{2}^{\prime}$ become the medians and $\mathrm{AA}_{1},{ }^{\prime} \mathrm{BB}_{1}, \mathrm{CC}_{1}^{\prime}$ the perpendiculars of the triangle $A B C$, the twelve-point conic again reducing to the nine-points circle (or twelve-points circle, as it might be called) of $\mathrm{ABC}$.

Going back to the general case, we might note other sets of collinear points and concurrent lines. This will appear in the next section, where the figure is considered from another point of view.

Meanwhile, we may note the set of six collinear points $D_{1} D_{2}^{\prime} M_{1} M_{2} D_{1}^{\prime} D_{2}$, where we have

$$
\begin{aligned}
& D_{2}^{\prime} M_{1}=\frac{1}{4} D_{2}^{\prime} D_{1}^{\prime} \text { as to the line } P_{1}^{\prime} Q_{1}^{\prime} R_{1}^{\prime} \\
& \mathrm{D}_{1}^{\prime} \mathbf{M}_{2}=\frac{1}{4} \mathrm{D}_{1}^{\prime} \mathrm{D}_{2}^{\prime} \quad " \quad, \quad " \quad, \quad \mathbf{P}_{2}^{\prime} \mathbf{Q}_{2}^{\prime} \mathbf{R}_{2}^{\prime} \\
& \mathrm{D}_{1} \mathrm{D}_{2}^{\prime}=\frac{1}{2} \mathrm{D}_{2}^{\prime} \mathrm{D}_{1}^{\prime}, " \quad " \quad, \quad \mathrm{P}_{1}^{\prime} \mathrm{Q}_{1}^{\prime} \mathbf{R}_{1}^{\prime} \\
& \mathrm{D}_{2} \mathrm{D}_{1}^{\prime}=\frac{1}{2} \mathrm{D}_{1}^{\prime} \mathrm{D}_{2}^{\prime} \quad " \quad, \quad, \quad, \quad \mathrm{P}_{2}^{\prime} \mathrm{Q}_{2}^{\prime} \mathbf{R}_{2}^{\prime} \text {. }
\end{aligned}
$$

The first statement of the four means, by analogy with the expression previously defined, "bisection of a line segment as to a line " that $M_{1}$ and the point of intersection of $D_{1} D_{2}$ with $P_{1}^{\prime} Q_{1}{ }^{\prime} R_{1}^{\prime}$ divide $D_{2}^{\prime} D_{1}^{\prime}$ in the anharmonic ratio $-\frac{1}{4}$. This implies, of course, that when $P_{1}^{\prime} Q_{1}^{\prime} R_{1}^{\prime}$ goes off to infinity $D_{2}^{\prime} M_{1}$ becomes actually $=\frac{1}{4} \mathrm{D}_{2}^{\prime} \mathrm{D}_{1}^{\prime}$.

We remark that five lines, consisting of a triad (the sides of the triangle $\mathrm{ABC}$ ) and a pair (the two transversals) determine uniquely a certain conic (the twelve-points conic).

Hence if any five straight lines be given, since they can be divided into a triad and a pair in ${ }^{5} \mathrm{C}_{2}=10$ different ways they determine a set of ten twelve-points conics. Into the relations existing between these ten conics I do not enter.

In place of determining the conic by a triangle and two transversals, we might of course let it be determined by the triangle and two points, viz., the "poles" of the transversals with respect to the triangle.

$\$ 5$.

The figure $A_{1} B_{1} C_{1} D_{1} A_{1}^{\prime} B_{1}^{\prime} C_{1}^{\prime} D_{1}^{\prime}$ is formed by three four-ray pencils with collinear apices $X_{1} Y_{1} Z_{4}$ intersecting so as to form a figure similar to a plane projection of a parallelepiped. We shall study it separately, generalize it, and apply it to a further generalization of the original figure. In this section, and the next following, we will for simplicity drop the suffixes of the letters. 


\section{1}

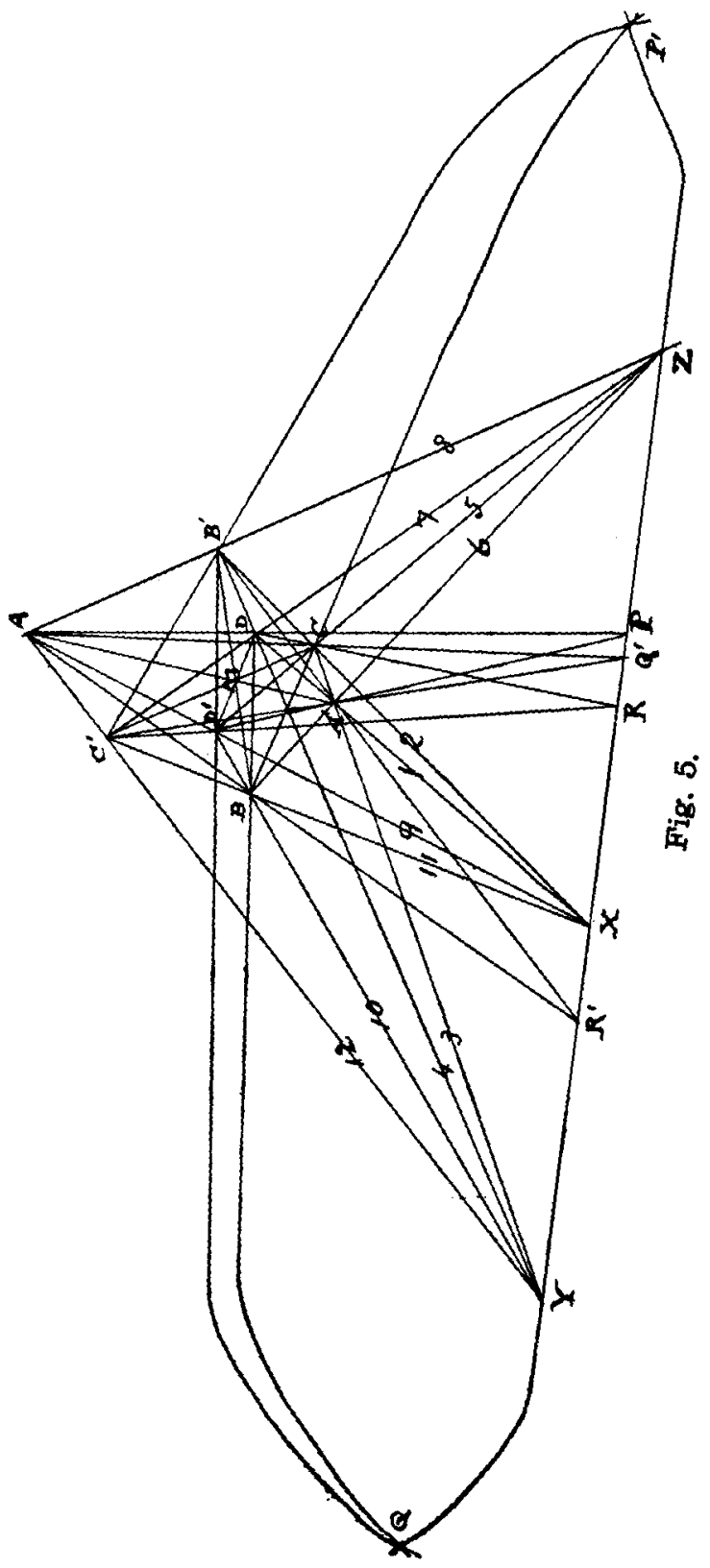


The figure we are now to consider may be constructed by following the order of drawing the lines indicated by the numerals on Fig. 5 .

Thus, choosing $\mathrm{X}$ and $\mathrm{X}$ and drawing any two pairs of lines 1,$2 ; 3,4$ through $\mathrm{X}$ and $\mathrm{Y}$ respectively, intersecting one another in $\mathrm{DB}^{\prime} \mathrm{CA}^{\prime}$, we then choose a point $\mathrm{Z}$ in $\mathrm{XY}$ and join it to $\mathrm{C}, \mathrm{A}^{\prime}, \mathrm{D}, \mathrm{B}^{\prime}$ by the lines $5,6,7,8$.

Then choose $\mathrm{D}^{\prime}$ in $\mathrm{ZC}$.

The line $\mathrm{XD}^{\prime}$ meets $\mathrm{ZB}^{\prime}$ in $\mathrm{A}$, while $\mathrm{YD}^{\prime}$ meets $\mathrm{ZA}^{\prime}$ in $\mathrm{B}$. Then YA and $\mathrm{XB}$ will concur with $\mathrm{ZD}$ in $\mathrm{C}^{\prime}$. This is easily proved, for as to $X Y Z$ we have, taking $\mathrm{C}^{\prime}$ as the intersection of $\mathrm{XB}$ and $\mathrm{ZD}, \mathrm{C}^{\prime} \mathrm{B} \equiv \mathrm{DA}^{\prime} \equiv \mathrm{B}^{\prime} \mathrm{C} \equiv \mathrm{AD}^{\prime}$. Hence by Prop. (i) $\mathrm{BD}^{\prime} \equiv \mathrm{C}^{\prime} \mathrm{A}$ as to $\mathrm{XYZ}$, i.e. $\mathrm{YA}$ produced passes through $\mathrm{C}^{\prime}$.

Since $\mathrm{C}^{\prime} \mathrm{A} \equiv \mathrm{A}^{\prime} \mathrm{C}$ it follows that $\mathrm{CA}^{\prime}$ and $\mathrm{AC}$ coneur with $\mathrm{XYZ}$ in a point $Q^{\prime}$.

Similarly $B^{\prime} C^{\prime}$ and $B C$ concur with $X Y Z$ in a point $P^{\prime}$.

\begin{tabular}{|c|c|c|c|c|c|c|c|c|}
\hline ", & $\mathbf{A}^{\prime} \mathrm{B}^{\prime}$ & " & $\mathrm{AB}$ & " & $"$ & $"$ & $"$ & " \\
\hline " & DA & $"$ & $\mathrm{D}^{\prime} \mathbf{A}^{\prime}$ & $"$ & $"$ & $"$ & $n$ & " \\
\hline "y & $\mathrm{DB}$ & $"$ & $D^{\prime} B^{\prime}$ & $"$ & "' & $"$ & $"$ & $"$ \\
\hline & $\mathrm{DC}$ & $"$ & $\mathrm{D}^{\prime} \mathrm{C}^{\prime}$ & $"$ & " & " & ", & ", \\
\hline
\end{tabular}

From Prop. (iii) it follows that $\mathrm{AA}^{\prime}, \mathrm{BB}^{\prime}, \mathrm{CC}^{\prime}, \mathrm{DD}^{\prime}$ all bisect one another as to $X Y Z$ in the same point $M$.

If we leave out two opposite points, say $\mathrm{DD}^{\prime}$, the remaining six, $\mathrm{AC}^{\prime} \mathrm{BA}^{\prime} \mathrm{CB}^{\prime}$ will lie on a conic in virtue of the converse of Pascal's Theorem, and this conic has obviously $M$ as the pole of XYZ.

The number of independent variables (freedoms) in the figure may be reckoned thus: $\mathrm{X}$ and $\mathrm{Y}$ have two freedoms each, $1,2,3,4$ have one freedom each.

$\mathrm{Z}, \mathrm{D}^{\prime}$ have one each. The other points and lines are then fixed. Thus we have a total of 10 freedoms.

We arrive at the same result by noting that a conic has 5 freedoms, while the choice of 5 points on it gives 5 more freedoms, the 6 th point being thereby fixed.

It is clear, too, that we may begin by choosing any 5 points (10 freedoms) out of the six $\mathrm{ACB} \mathrm{A}^{\prime} \mathrm{C}^{\prime} \mathrm{B}^{\prime}$, after which the 6th point and $D^{\prime} X Y Z P Q R P^{\prime} Q^{\prime} R^{\prime}$ are determined. 
The number of different ways they can be determined for a given set of 5 points is $5 \times 3$, for this is the number of ways in which we can single out one of the 5 points and arrange the other 4 in pairs.

$$
\$ 6 .
$$

If we remove the restriction that $\mathrm{XYZ}$ should be collinear, then one freedom is added to the figure, bringing the total up to 11. In this case Pascal's Theorem does not apply, and the six points do not necessarily lie on a conic.

The generalized figure (which is to be supplied by the reader) may be studied by means of a three-dimensional construction as follows :

Begin with the complete quadrilateral consisting of the triangle $\mathrm{ZB}^{\prime} \mathrm{D}$ and its transversal $\mathrm{YC}^{\prime} \mathrm{A}$, having $\mathrm{U}^{\prime} \mathrm{PP}^{\prime}$ as its diagonal triangle, $\mathrm{U}^{\prime}$ being the intersection of $\mathrm{DA}$ and $\mathrm{B}^{\prime} \mathrm{C}^{\prime}, \mathrm{P}$ that of $\mathrm{DA}$ and $\mathrm{YZ}, \mathrm{P}^{\prime}$ that of $\mathrm{YZ}$ and $\mathrm{B}^{\prime} \mathrm{C}^{\prime}$.

Let $X$ be an arbitrary point outside the plane of $\mathrm{AB}^{\prime} \mathrm{DC}^{\prime} \mathrm{YZ}$. Take a plane through $\mathrm{YZ}$ and let it cut the lines $\mathrm{XA}, \mathrm{XB}^{\prime}, \mathrm{XD}$, $\mathrm{XC}^{\prime}$ in $\mathrm{D}^{\prime} \mathrm{C} \mathrm{A}^{\prime} \mathrm{B}$ respectively. We thus get a pair of quadrilaterals triply in perspective. To prove this it is only necessary to show that $\mathrm{BD}^{\prime}$ passes through $\mathrm{Y}$, etc.

Now $\mathrm{BD}^{\prime} \mathrm{Y}$ is the intersection of the planes $\mathrm{YZA}^{\prime}$ and $\mathrm{XYC}$, so that $B, D^{\prime}, Y$ are collinear. Similarly $A^{\prime} C$ passes through $Y$, and of course $\mathrm{DB}^{\prime}$ and $\mathrm{C}^{\prime} \mathrm{A}$ do so.

In like manner we can show that $\mathrm{DC}^{\prime}, \mathrm{D}^{\prime} \mathrm{C}, \mathrm{BA}^{\prime}, \mathrm{B}^{\prime} \mathrm{A}$ are concurrent in $Z$.

The solid figure $\mathrm{AD}^{\prime} \mathrm{BC}^{\prime} \mathrm{DA}^{\prime} \mathrm{CB}^{\prime}$ is topologically the same as a cube or parallelepiped, but having 3 sets of 4 edges concurrent instead of parallel.

Let $\mathrm{DA}, \mathrm{B}^{\prime} \mathrm{C}^{\prime}$ meet in $\mathrm{U}^{\prime} ; \mathrm{D}^{\prime} \mathrm{A}^{\prime}, \mathrm{BC}$ in $\mathrm{U}$.

$$
\begin{aligned}
& \text { " } \mathrm{DB}, \mathrm{C}^{\prime} \mathrm{A}^{\prime} \quad \text { " } \mathrm{V}^{\prime} ; \mathrm{D}^{\prime} \mathrm{B}^{\prime}, \mathrm{CA}, \mathrm{V} \text {. } \\
& \text { "DC, } A^{\prime} B^{\prime} \quad, \quad, W^{\prime} ; D^{\prime} C^{\prime}, A B \text {, W. }
\end{aligned}
$$

The planes $\mathrm{XA}^{\prime} \mathrm{DAD}, \mathrm{XCB}^{\prime} \mathrm{C}^{\prime} \mathrm{B}$ both contain $\mathrm{U}, \mathrm{U}^{\prime}, \mathrm{X}$, hence $\mathrm{U}, \mathrm{U}^{\prime}, \mathrm{X}$ are collinear. So are $\mathrm{V}, \mathrm{V}^{\prime}, \mathrm{Y}$ and $\mathrm{W}, \mathrm{W}^{\prime}, \mathrm{Z}$.

Let $D B$ meet $X Z$ in $Q$ and $D C$ meet $X Y$ in $R$. The three

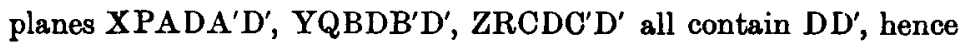
they have $\mathrm{DD}^{\prime}$ as common section, and $\mathrm{DD}^{\prime}$ will meet the plane 
$X Y Z$ in a point $\delta$, which will be the point of concurrence of the 3 lines $X P, Y Q, Z R$, in which these three planes intersect the plane XYZ.

Thus $\mathrm{AA}^{\prime}, \mathrm{BB}^{\prime}, \mathrm{CC}^{\prime}$ all meet $\mathrm{DD}^{\prime}$, since each of these lies in one of the planes of which $\mathrm{DD}^{\prime}$ is the common intersection. Again $\mathrm{BB}^{\prime}$ and $\mathrm{CC}^{\prime}$ lie in the plane $\mathrm{XBC}^{\prime} \mathrm{CB}^{\prime}$, and therefore intersect one another, hence their point of intersection, $M$, must lie on DD'. $^{\prime}$.

In other words, $M$ is the common point of the three planes $\mathrm{YQBDB}^{\prime} \mathrm{D}^{\prime}$, ZRCDC' $\mathrm{D}^{\prime}$, $\mathrm{XBC}^{\prime} \mathrm{CB}^{\prime}$, so that their mutual intersections, $\mathrm{BB}^{\prime}, \mathrm{CC}^{\prime}, \mathrm{DD}^{\prime}$ are concurrent in $\mathrm{M}$. Similarly $\mathrm{AA}^{\prime}$ cuts $\mathrm{DD}^{\prime}$ where it meets $\mathrm{CC}^{\prime}$, i.e. at $\mathrm{M}$.

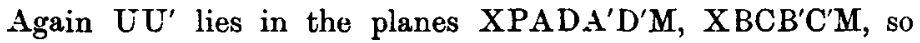
that $\mathrm{U}, \mathrm{U}^{\prime}, \mathrm{M}, \mathrm{X}$ are collinear. So are $\mathrm{V}, \mathrm{V}^{\prime}, \mathrm{M}, \mathrm{Y}$; and $\mathrm{W}, \mathrm{W}^{\prime}, \mathbf{M}, \mathrm{Z}$.

$U U^{\prime}$ and $W^{\prime} W^{\prime}$ being in one plane, $U W^{\prime}$ and $U^{\prime} W$ meet one another; but $U^{\prime} W$ lies in the plane PADBQ and $U W^{\prime}$ in the plane $R C D B Q$, therefore they meet in BDQ. But $U U^{\prime}$ and $W W^{\prime}$ lie in the plane $\mathrm{XMZ} \therefore \mathrm{UW}^{\prime}$ and $\mathrm{U}^{\prime} \mathrm{W}$ lie in that plane and meet $B D Q$ where it meets that plane, i.e. in $Q$.

\begin{tabular}{|c|c|c|c|c|c|}
\hline Thus & $\mathrm{UW}^{\prime}$ & and & $U^{\prime} W^{\prime}$ & neet & \\
\hline Similarly & $\mathrm{UV}^{\prime}$ & , & $\mathbf{C}^{\prime} \mathbf{V}$ & , & $"$ \\
\hline & $\mathbf{V} \mathbf{W}^{\prime}$ & " & $\mathbf{V}^{\prime} \mathbf{W}$ & $"$ & \\
\hline Also & VW & $"$ & $V^{\prime} W^{\prime}$ & ", & \\
\hline " & WU & $"$ & $\mathbf{W}^{\prime} \mathbf{U}^{\prime}$ & $"$ & \\
\hline$"$ & UV & $"$ & $\mathrm{U}^{\prime} \mathrm{V}^{\prime}$ & $"$ & " \\
\hline
\end{tabular}

where $\mathrm{P}^{\prime}$ is the point of intersection of $C B$ and $C^{\prime} B^{\prime}$ with $Y Z$, etc.

$P^{\prime}, Q^{\prime}, R^{\prime}$ are collinear since they are on the intersections of two planes $C^{\prime} U^{\prime} B^{\prime} W^{\prime} A^{\prime} V^{\prime}$ and $A W B U C V$. Hence also these planes and the plane $X Y Z$ have $P^{\prime} Q^{\prime} R^{\prime}$ as common intersection.

Also $B^{\prime} V^{\prime}$ and $B V$ which lie in the plane $\mathrm{YB}^{\prime} D V^{\prime} B Q D^{\prime} V$ (and respectively in the planes $C^{\prime} U^{\prime} B^{\prime} W^{\prime} A^{\prime} V^{\prime}$ and $A W B U C V$ ) must intersect where $P^{\prime} Q^{\prime} R^{\prime}$ meets this plane, i.e. where $Y Q$ meets $\mathbf{P}^{\prime} \mathbf{Q}^{\prime} \mathbf{R}^{\prime}$, say in $\mathbf{Y}^{\prime}$.

Similarlly $\mathbf{C}^{\prime} W^{\prime}$ and $C W$ concur with $P^{\prime} Q^{\prime} R^{\prime}$ in $Z^{\prime}$.

$$
\text { " } \mathbf{A}^{\prime} \mathbf{U}^{\prime}, \mathbf{A U} \quad, \quad ", \quad, \mathbf{X}^{\prime} \text {. }
$$

Again $\mathrm{A}^{\prime} \mathrm{U}^{\prime}, \mathrm{B}^{\prime} \mathrm{V}^{\prime}, \mathrm{C}^{\prime} \mathrm{W}^{\prime}$ concur in $d$ and $\mathrm{AU}, \mathrm{BV}, \mathrm{C}^{\prime} \mathrm{W}^{\prime}$ in $d^{\prime}$, which both lie on $\mathrm{DM}^{\prime} \delta$. 
Note that $\delta$ is the point in which $\mathrm{DD}^{\prime}$ meets the plane XYZ. Similarly we have points $\alpha, \beta, \gamma$ which are the intersections of $\mathrm{AA}^{\prime}, \mathrm{BB}^{\prime}, \mathrm{CC}^{\prime}$ with the same plane.

$$
\begin{aligned}
& \text { a lies in } \mathrm{XPX}^{\prime} \text { in } \mathrm{YQ}^{\prime} \text { and in } \mathrm{ZR}^{\prime}
\end{aligned}
$$

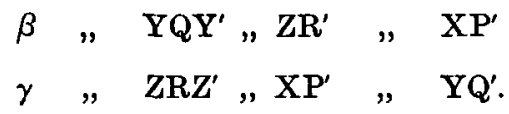

Our figure has been developed with special regard to $\mathrm{DD}^{\prime}$, but it might equally have been developed with special regard to $A A^{\prime}$, in which case $M X Y Z P^{\prime} Q^{\prime} R P Q R^{\prime}$ would take the places of MXYZ PQR $P^{\prime} Q^{\prime} R^{\prime}$, etc. It might also be developed with special reference to $\mathrm{BB}^{\prime}$ or $\mathrm{CC}^{\prime}$.

We observe that a doubly infinite family of quadric surfaces exists each member of which passes through $A B C D A^{\prime} B^{\prime} C^{\prime} D^{\prime}$ (since these eight points are common to three pairs of planes), and has $\mathbf{M}$ as the pole of the plane $\mathrm{XYZ}$.

Note the plane figure consisting of the triangle $X Y Z$ and the transversal $P^{\prime} Q^{\prime} R^{\prime}$. These determine $P Q R X^{\prime} Y^{\prime} Z^{\prime} a \beta \gamma \delta$ in the same plane; or if $P Q R$ and its transversal $P^{\prime} Q^{\prime} R^{\prime}$ be taken, they determine the diagonal triangle $X Y Z$ and also the points $\mathrm{X}^{\prime} \mathbf{Y}^{\prime} \mathbf{Z}^{\prime} \alpha \beta \gamma \delta$.

Now let us consider a plane passing through $\mathrm{A}^{\prime} \mathrm{B}^{\prime} \mathrm{C}^{\prime}$. It passes also through $P^{\prime} Q^{\prime} R^{\prime}$. The lines $P^{\prime} A^{\prime}, Q^{\prime} B^{\prime}, R^{\prime} C^{\prime}$ will form a triangle which we may name $A_{0} B_{0} C_{0}$ and it is clear that $A_{0}$ lies in $\mathrm{AD}^{\prime}, \mathrm{B}_{0}$ in $\mathrm{BD}^{\prime}$, and $\mathrm{C}_{0}$ in $\mathrm{CD}^{\prime}$.

The solid figure studied in this section can be projected on any plane (the plane of the paper), and the properties obtained above by solid geometry might be worked out by the aid of the theorems of Ceva and Menelaus and the theory of anharmonic ratio.

$$
\$ 7 .
$$

Let us now suppose the figure of $\S 6$ to be projected on the plane of the paper, the projections of all the points being denoted by corresponding letters with suffix 1 , with the exception of $\mathrm{A}_{0} \mathrm{~B}_{0} \mathrm{C}_{0}$, whose projections will be denoted by $\mathrm{ABC}$. 


\begin{tabular}{|c|c|c|}
\hline Let & $\mathrm{B}_{1}{ }^{\prime} \mathrm{C}_{1}^{\prime}$ meet & $\mathrm{BC}$ \\
\hline " & $\mathbf{C}_{1}^{\prime} \mathbf{A}_{1}^{\prime}$ & $\mathbf{C A}$ \\
\hline " & $\mathbf{A}_{1}^{\prime} \mathbf{B}_{\mathbf{1}}^{\prime}$ & $\mathrm{AB}$ \\
\hline " & $\mathbf{A A _ { 1 }} \mathbf{D}_{1}^{\prime} "$, & $\mathrm{BC}$ \\
\hline$"$ & $\mathrm{BB}_{1} \mathrm{D}_{1}^{\prime} \quad "$ & $\mathrm{CA}$ \\
\hline , & $\mathrm{CC}_{1} \mathrm{D}_{1}^{\prime}$, & $\mathrm{AB}$ \\
\hline
\end{tabular}

Let $\mathrm{D}_{2}^{\prime}$ be the point of concurrence of $\mathrm{AA}_{1}^{\prime}, \mathrm{BB}_{1}^{\prime}$, and $\mathrm{CC}_{1}^{\prime}$.

Through $P_{2}^{\prime}$ draw an arbitrary line meeting $\mathrm{BD}_{2}^{\prime}$ in $\mathrm{B}_{2}$ and $\mathrm{CD}_{2}^{\prime}$ in $\mathrm{C}_{2}$.

Then $Q_{2}^{\prime} C_{2}$ and $R_{2}^{\prime} B_{2}$ will meet in $A_{2}^{\prime}$ in a point $A_{2}$ $\mathrm{B}_{2} \mathrm{C}_{2}^{\prime}, \mathrm{B}_{2}^{\prime} \mathrm{C}_{2}$, and $\mathrm{A}_{2} \mathrm{D}_{2}^{\prime}$ will concur in a point $\mathrm{X}_{2}$ $\mathrm{C}_{2} \mathrm{~A}_{2}^{\prime}, \mathrm{C}_{2}^{\prime} \mathrm{A}_{2}, \quad, \quad \mathrm{~B}_{2} \mathrm{D}_{2}^{\prime} \quad$ " $, \quad, \quad " \mathbf{Y}_{2}$ $\mathrm{A}_{2} \mathrm{~B}_{2}^{\prime}, \mathrm{A}_{2}^{\prime} \mathrm{B}_{2} \quad, \mathrm{C}_{2} \mathrm{D}_{2}^{\prime} \quad " \quad$ ",$\quad \mathrm{Z}_{2}$.

$\mathrm{X}_{2} \mathrm{~A}_{2}^{\prime}, \mathrm{Y}_{2} \mathrm{~B}_{2}^{\prime}$ and $\mathrm{Z}_{2} \mathrm{C}_{2}^{\prime}$ will concur in a point $\mathrm{D}_{2}$, and the figure may be further developed until there is a point with suffix 2 corresponding to every point with suffix 1 .

We have now arrived at a generalization of the figure of $\$ 4$, since the points $X_{1} Y_{1} Z_{1}$ are no longer restricted to be collinear, nor are $X_{2} Y_{2} Z_{2}$ so restricted. The twelve points

$$
A_{1} B_{1} C_{1} A_{1}^{\prime} B_{1}^{\prime} C_{1}^{\prime} A_{2} B_{2} C_{2} A_{2}^{\prime} B_{2}^{\prime} C_{2}^{\prime}
$$

no longer necessarily lie on a conic.

To get the figure of $\$ 4$ as a special case of that now considered we must suppose the projection of the solid figure to be from a centre in the intersection of the planes $X_{1} Y_{1} Z_{1}$ and $X_{2} Y_{2} Z_{2}$, so that each of these planes will project into a straight line. The points $X_{1} Y_{1} Z_{1} P_{1} Q_{1} R_{1} P_{1}^{\prime} Q_{1}^{\prime} R_{1}^{\prime} X_{1}^{\prime} Y_{1}^{\prime} Z_{1}^{\prime} a_{1} \beta_{1} \gamma_{1} \delta_{1}$ etc., in fact all in the plane $X_{1} Y_{1} Z_{1}$ become collinear, as also $X_{2} Y_{2} Z_{2} P_{2} Q_{2} R_{2}$, etc., and the twelve points $A_{2} B_{1} C_{1} A_{1}^{\prime} B_{1}^{\prime} C_{1}^{\prime} A_{2} B_{2} C_{2} A_{2}^{\prime} B_{2}^{\prime} C_{2}^{\prime}$ would lie on a conic, as in $\$ 4$.

If we wish to get the generalized figure of the present section by a plane construction we can begin as in $\$ 4$ with the triangle $A B C$, and proceed as in $\$ 4$ to get the points

$P_{1}^{\prime} Q_{1}^{\prime} R_{1}^{\prime} A_{1}^{\prime \prime} B_{1}^{\prime \prime} C_{1}^{\prime \prime} A_{1}^{\prime} B_{1}^{\prime} C_{1}^{\prime} D_{2}^{\prime} P_{2}^{\prime} Q_{2}^{\prime} R_{2}^{\prime} A_{2}{ }^{\prime \prime} B_{2}{ }^{\prime \prime} C_{2}{ }^{\prime \prime} A_{2}^{\prime} B_{2}^{\prime} C_{2}^{\prime} D_{1}{ }^{\prime}$, 


\section{7}

but instead of taking $X_{1}$ as intersection of $A_{A_{2}^{\prime}}$ with $P_{1}^{\prime} Q_{1}^{\prime} R_{1}^{\prime}$ let $\mathrm{X}_{1}$ be an arbitrary point in $\mathrm{AA}_{2}^{\prime}$. Then let $\mathrm{X}_{1} \mathrm{~B}_{1}^{\prime}$ and $\mathrm{X}_{1} \mathrm{C}_{1}^{\prime}$ cut $\mathrm{C}_{0} \mathrm{C}_{2}^{\prime}$ and $\mathrm{B}_{0} \mathrm{~B}_{2}^{\prime}$ in $\mathrm{C}_{1}$ and $\mathrm{B}_{1}$ which will be collinear with $\mathrm{P}_{1}^{\prime}$. Then $Z_{1}$ and $Y_{1}$ are the intersections of $B_{1} A_{1}{ }^{\prime}$ and $C_{1} A_{1}^{\prime}$ with $X_{1} Q_{1}^{\prime}$ and

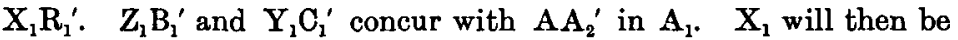
the point of concurrence of $B_{1} C_{1}^{\prime}, C_{1} B_{1}^{\prime}, A_{1} D_{1}^{\prime}$ and $A_{1}^{\prime} D_{1}$; and similarly for $Y_{1}$ and $Z_{1}$. $P$ will be the point of concurrence of $A_{1} D_{1}, A_{1}^{\prime} D_{1}^{\prime}$ and $Y_{1} Z_{1}$, and similarly for $Q$ and $R$.

The other points with suffix 2 can then be constructed as in the first part of the present section, in which it is to be noted there is one arbitrary element which may be taken as the point $\mathrm{B}_{2}$ in $\mathrm{BD}_{2}^{\prime}$.

Thus on the whole the figure of the present section has two additional freedoms as compared with that of $\$ 4$.

Note.-The last section (\$7) has been re-written and made more complete since the paper was read. 